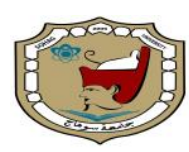

\title{
Carbapenem Resistance In Enterobacteriaceae
}

\author{
Hadir A. Elmahllawy ${ }^{1}$, Hasnaa A. Abo-Elwafa ${ }^{2}$, Laila M. Yousif ${ }^{2}$, \\ Radwa M. Farag ${ }^{2}$.
}

\author{
1- Clinical Pathology department, National Cancer Institute,, Cairo \\ University
}

2- Clinical Pathology department, Faculty of Medicine, Sohag University

\begin{abstract}
Carbapenem resistance is now a public health concern worldwide. Carbapenem is considered the last resort antimicrobial drug used for treatment of hospital careassociated infection and community-acquired infection that resistant to all other $\beta$ lactam drugs. There are different mechanisms by which bacteria become resistant to carbapenem drug including efflux pump, porin mutation and production of carbapenemase enzyme that hydrolyze the drug. Carbapenemase enzymes were identified largely in different members of the Enterobacteriaceae family which is a gram-negative bacteria responsible for a variety of infectious diseases and this was associated with increased morbidity and mortality rate worldwide. Many Risk factors were found to be associated with increased susceptibility to develop carbapenem resistance that should be searched for to prevent further spread of resistance. Various phenotypic and genotypic tests are used to detect carbapenemase production with different sensitivity and specificity. The current state of carbapenem resistance is well identified in many parts of the world while in other places such as sub-Saharan Africa, this is not well known.
\end{abstract}

Keywords: carbapenem,resistance, phenotypic test,

\section{Introduction}

Carbapenem-resistant Enterobacteriaceae (CRE) is now a public health problem worldwide (1). In the early 1990s., the first discovered carbapenem resistance-in Enterobacte-riaceae was published and in 2001, the first Klebsiella pneumoniae carbapenemase (KPC) producing Enterobact-eriaceae was reported. Thereafter, CRE has disseminated globally (2).

\section{Carbapenems}

Carbapenems are bactericidal $\beta$-lactam antibiotics used in the management of severe infections caused by bacteria that produce extended-spectrum $\beta$-lactamase with high efficacy (3). Meropenem, imipenem, ertapenem and dorip- enem are a few examples of carbapenem in use worldwide. Recently several mechanisms of resist-ance emerged as $\beta$-lactamases that hydrolyze carbapenem leaving narrow therapeutic options (4).

\section{Mechanism of action}

The $\beta$-lactam family of antibiotics that includes; penicillins, cephalosporins, monobactam and carbapenems shares common structure and mechanism of action. After entering through porins to the periplasmic space they inhibit transpeptidases. These enzymes enhance peptide cross-links during the synthesis of the cell wall. The similarity of $\beta$-lactam toD-alanyl-D-alanine (a res- 
id-ue used in the formation of peptidoglycan) facilitate their binding to the transpeptidases. Carbapenem binds to transpeptidases, which causes them to lose their catalytic activity. This inhibits peptidoglycan polymers synth-esis by inhibiting the formation of crosslinks between them that causes a buildup of new peptidoglycan precu-rsors without cross-linkages. This weak peptidoglycan in addition to the continued activity of autolysins, which destroys peptide bonds of peptid-oglycan, disturbs the cell wall and causes osmotic rupture of the cell (5).

\section{Carbapenem resistannce Enteroba- cteriaceae (CRE)}

CRE are Gram-negative bacteria resist carbapenem antibiotics that considered the drugs of last resort by producing enzyme called carbapenemase that hydrolyzes the antibiotic molecule. Experts have referred to CRE as "nightmare bacteria (6).

In 2013, CDC report that half of the patients who get bloodstream infections can be killed up by these bacteria (7)

Enterobacter cloacae (E. cloacae), as well as other Enterobacteriaceae, is a commensal organism present in the intestine. Infections caused by $\mathrm{E}$. cloacae arranged as the third among all the Enterobacteriaceae (8).

Chromosome mediated AmpC $\beta$-lactamase is produced by Enterobacter cloacae and Enterobacter cloacae have resistance to amoxicillin/clavulanic, ampicillin, first and second-generation cephalosporin and cephamycin. So, carbapenems are used in the treatment that leads to the emergence of multi- drug resistance rapidly under antibiotic selection pressure. In many countries such as India, Australia, Spain, China and the United States, carbapenemresistant E. cloacae infections have been documented $(\mathbf{9}, \mathbf{1 0}, \mathbf{1 1})$.

The main mechanisms for carbapenem resistance in $\mathrm{E}$. cloacae is a decrease in membrane permeability and producing carbapenemases. Also, combinations of either AmpC or ESBL and mutation of porins are other important mechanisms (12).

\section{Mechanisms of carbapenem resistance:}

The 3 main mechanisms for carbapenem resistance are efflux pumps, porin mutations and enzyme production (13).

1. Efflux pumps that actively transport carbapenem outside of the cell wall have been observed in resistant bacteria (14).

2. Porins mutation or loss of it that prevents the entrance of carbapenem into the periplasm space (15).

3. Carbapenemases enzymes which are a form of $\beta$-lactamase, destroy the $\beta$-lactam ring which is an important component of $\beta$-lactam drugs that bound to transpeptidases, is produced by Enterobacteriaceae. Carbap-enemases are classified according to the structure of the enzyme and the mechanism of the $\beta$-lactam hydrolysis into several classes (16).

In general there are two subgroups for CRE: carbapenemase-producing CRE (CP-CRE) and non-carbapenemaseproducing $\mathrm{CRE}$ (non-CP-CRE) (Figure 1) (17). 


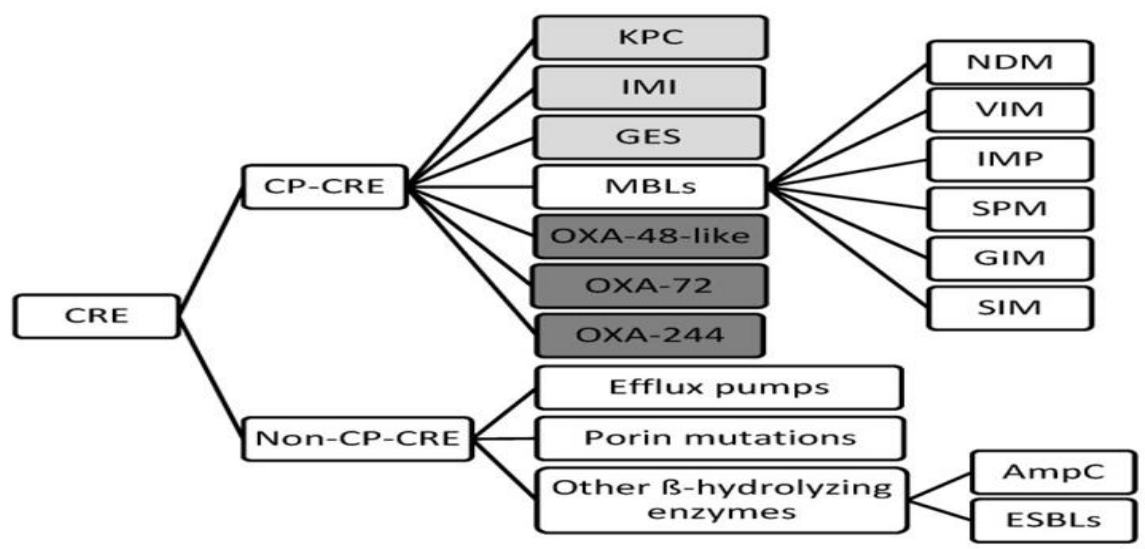

Figure 1: Classification of carbapenem resistance mechanisms in CRE.

AmpC : Type C ampicillinase * CP : Carbapenemase producing * CRE : Carbapenem-resistant Enterobacteriaceae *

ESBLs :Extended-spectrum ß-lactamase * GES :Guiana extended-spectrum ß-lactamase * GIM : German imipenemase * IMI: Imipenem-hydrolyzing B-lactamase* IMP: Imipenemresistant Pseudomonas carbapenemase*

KPC : Klebsiella pneumoniae carbapenemase* MBLs : Metallo-beta-lactamases * NDM: New Delhi metallo- $\beta$-lactamase* OXA : Oxacillinase * SIM : Seoul imipenemase * SPM : Sao Paulo metallo- $\beta$-lactamase*VIM: Verona integron-encoded metallo- $\beta$-lactamase

\section{Classification of carbapenemases}

Enzymes that cause carbapenem classes according to Ambler resistance are divided into three main classification (18) (Table 1).

\begin{tabular}{|l|l|l|l|}
\hline Carbapenemase & KPC & MBLs (NDM, VIM, IMP) & OXA-48 \\
\hline Ambler class & A & B & D \\
\hline $\begin{array}{l}\text { Substrates of } \\
\text { hydrolysis }\end{array}$ & $\begin{array}{l}\text { whole } \beta \text { - } \\
\text { lactams }\end{array}$ & $\begin{array}{l}\text { whole } \beta \text {-lactams except for } \\
\text { aztreonam }\end{array}$ & $\begin{array}{l}\text { Penicillins and } \\
\text { carbapenems }\end{array}$ \\
\hline $\begin{array}{l}\text { classic } \beta \text {-lactamase } \\
\text { inhibitors }\end{array}$ & Minimally & No & No \\
\hline Avibactam inhibition & Yes & No & Yes \\
\hline $\begin{array}{l}\text { Common species } \\
\text { in Enterobacteriaceae }\end{array}$ & $\begin{array}{l}\text { K.pneumoniae } \\
\text { E.coli, } \\
\text { Enterobacter s } \\
\text { pp. }\end{array}$ & $\begin{array}{l}\text { NDM: K. pneumoniae, E. } \\
\text { coli VIM: K. } \\
\text { pneumoniae IMP: K. } \\
\text { pneumonia }\end{array}$ & K. pneumia \\
\hline
\end{tabular}

Table 1: Classification of common carbapenemases in Enterobacteriaceae.

\section{Class A $\beta$-lactamases}

These enzymes either chromosomally encoded as not metalloenzyme carbapenemase A (NmcA), Imipenem-hydrolysing $\beta$-lactamase (IMI), Serratia fonticola carbapenemase (SFC) and Serratia marcescens enzyme (SME), or plasmid encoded as Klebsiella pneumoniae carbapenemase (KPC), Guiana extended-spectrum (GES) and its derivative (GES-1 - GES-20), but all are partially inhibited by clavulanic acid
$(19,20)$. Class A carbapenemases are serine carbapenemases which mean that serine is required for hydrolysis of $\beta$ lactams that present at the active site of these enzymes (21).

In addition to $\mathrm{K}$. pneumoniae isolates, other members of Enterobacteriaceae have been found to produce KPC including Klebsiella oxytoca, Escherichia coli, Proteus mirabilis, Salmonella enterica, Citrobacter freundii, Serratia marcescens and Enterobacter species (Ente- 
robacter aerogens and Enterobacter cloacae) $(\mathbf{2 2}, \mathbf{2 3})$.

\section{Class B metallo- $\beta$-lactamases (MBL)}

NDM (New Delhi Metallo- $\beta$-lactamase), GIM (German imipenemase), IMP carbapenemases (Imipenem-resistant Pseudomonas), SIM (Seoul imipenemase) and VIM (Verona integronencoded Metallo- $\beta$-lactamase) are members of Metallo- $\beta$-lactamase families. These enzymes are encoded by genes present within integron structures and gene cassettes. Zinc at the active site of these enzymes is required for hydrolysis of carbapenem drugs hence, these enzymes can be inhibited by ethylenediaminetetraacetic acid (EDTA), a chelator of $\mathrm{Zn} 2+$ (24).

\section{Class D $\beta$-lactamases}

These carbapenemases include OXA enzyme type and the blaOXA genes are present on both chromosomes and plasmids. These enzymes are serine- $\beta$ lactamases and poorly inhibited by clavulanic acid and EDTA. In 1985, OXA $\beta$-lactamase was first described and the enzyme was isolated from patients in Scotland infected by Acinetobacter baumannii (25). There is wide geographic dissemination of OXA carbapenemase due to increased activity of OXA carbapenemases by upstream elements that control gene expression and also increase the strength of their promoters by insertions in the vicinity of these genes and hence increase resistance (21). OXA carbapenemases can mutate rapidly and they were reported frequently in the Enterobacteriaceae family which is a major public health problem worldwide (26). The true prevalence rates of OXA-48 are difficult to estimate and OXA-48 producers are difficult to identify due to their point mutant analogs with extendedspectrum $\beta$-lactamase (24).

\section{Risk factors:}

Primary transmission places for CRE infections are hospital. Hospital admissions due to CRE from long-term acute care facilities (LTAC) or transferred from another hospital account for $75 \%$. A significantly higher incidence of colonization and infection was found in patients admitted from LTAC facilities comparing with other hospitalized patients. A study in 2012, has been stated that over $30 \%$ of the patients with LTAC exposure was found to be colonized with CRE $(\mathbf{2 7}, \mathbf{2 8})$

High incidence and prevalence of CRE infections were reported in countries with antibiotic abuse where antibiotics are obtainable without a prescription. Because of limited antibiotic use in Japan, only $6.4 \%$ of healthy populations found to carry ESBL producing strains whereas in Thailand and Egypt, 58.4\%, and $63.3 \%$ of the healthy population respectively were colonized because of the availability of antibiotics without prescription (28). Other Risk factors for CRE infection include mechanical ventilation, abuse of antibiotics, diabetes and compromised immune response Suboptimal maintenance practices includes inadequate cleaning and disinfection of medication cabinets, patient rooms, and medical equipment, that are used for both non-CRE patients and CRE are a cause of CRE (29). It has been found that exposure to different types of antibiotics (carbapenems, quinolones, glycopeptides, and $\beta$-lactams), organ/stem cell transplantation, mechanical ventilation, long time of hospitalization, ICU admission, surgery, urinary catheter, nasogastric catheter and central venous catheterization are risk factors for CRE (30). Decreases in the CRE rate was found in the hospital in which contact precaution for patients infected with Gram-negative bacilli was taken. Thus, it is important to find out the source of infection and patients who have any risk factor of infection and the main focus in treating patients at high 
risk should be to prevent CRE infection $(31,32)$.

\section{Identification of carbapenemases:}

Many trials have been come out to detect the production of carbapenemases in bacteria. They divided into phenotypic and genotypic tests.

\section{Phenotypic tests:}

\section{Modified Hodge Test}

According to this test, the tenth dilution of 0.5 McFarland suspension of $E$ Coli ATCC is inoculated on MuellerHinton agar plate and an imipenem $\operatorname{disc}(10-\mu \mathrm{g})$ is put in the center of the plate. Then the carbapenem-resistant strain is spreaded from the edge of the disc to the periphery of the plate therefore to form a straight line of inoculum. The plate is incubated overnight. Imipenem is hydrolyzed and the susceptible E. coli grow toward the disc, making a cloverleaf-like appearance if carbapenemase is released by the test strain. The MHT had been recommended as a confirmation test because of its high sensitivity and specificity in detecting carbapenemases $(33,34)$.

\section{Carbapenem Inactivation Method}

Firstly, a meropenem $(10-\mu \mathrm{g})$ disc is incubated with the test strain for 2 hours at $35^{\circ} \mathrm{C}$. Then, the meropenem disc is removed from the solution and put on a Mueller Hinton agar plate inoculated with sensitive E. coli strain. During the initial incubation the meropenem disc would have been inactivated, allowing for the growth of E. coli, making it appear as if the susceptible E. coli is resistant if carbapenemase is released but if no carbapenemase is released, an inhibition zone is formed. Because of its low cost and does not need special skills, the Clinical and Laboratory Standards Institute (CLSI) recommended this test as one of the confirmation tests of CPE. This method can be used for the detection of IMP,
KPC, OXA-48 and Metallo-beta-lactamases such as NDM and VIM with sensitivity about $99 \%(\mathbf{3 5}, \mathbf{3 6})$.

\section{Combined disc Test}

The chelating activity of ethylenediaminetetraacetic acid (EDTA) to zinc metal let it to be useful in detecting MBL enzymes as these enzymes need zinc for their activities. EDTA is added to a carbapenem disc, then is put on a plate inoculated with the suspected carbapenem resistance strain. A larger zone of inhibition around the EDTA/carbapenem disc compared with the carbapenem disc without EDTA indicates that MBL is present $(\mathbf{3 7}, \mathbf{3 8})$.

\section{Boronic Acid Inhibition Test}

It is known that boronic acid compounds excellent inhibitors of class $C \beta$ lactamases. Recently, these compounds can be used for inhibition of class A carbapenemases. According to this test, 2-aminophenyl boronic acid (300 or $400 \mu \mathrm{g}$ ) is added to an ertapenem or meropenem disc. If $\mathrm{KPC}$ is present, there will be an increase in the zone of inhibition of $5 \mathrm{~mm}$ or greater compared with the ertapenem or meropenem disc alone $(39,40)$.

\section{Carba NP test}

This test is a rapid colorimetric test depend on the detection of $\mathrm{pH}$ changes that occur when imipenem is hydrolyzed by carbapenemases. The Carba NP test is highly sensitive in identifying MBL and KPC enzymes but difficulty identifying the activity of OXA-48 and GES-5. It is one of the phenotypic tests recommended by CLSI for the detection of carbapenemases (41).

\section{Molecular tests}

1. Polymerase Chain Reaction(PCR) Various types of PCR as conventional, real-time, and multiplex PCR can be used for the detection of carbapenemase with high sensitivity and specificity (42). 


\section{Verigene}

Verigene is a non-amplified test depends on nucleic acid extraction from positive blood cultures, microarraybased using capture and probes for detection. The process needs about 5 minutes for sample preparation and 2 hours for a run and so it is very useful in the rapid detection of various carbapenemase genes $(\mathbf{4 2}, \mathbf{4 3})$.

\section{BioFire FilmArray}

It is an automated multiplex PCR that although firstly used for rapid detection of bacteremia and fungemia, now it can be used to detect the presence of carbapenemase genes as blaKPC. The sample preparation takes about 23 minutes and a turnaround time of 1 hour. It has $100 \%$ sensitivity and specificity in the detection of blaKPC but its cost is high which limits its use (44).

\section{Gene Xpert}

The detection of bla KPC, bla NDM, bla VIM, bla IM $\mathrm{P}$, and bla OXA-48 can be achieved by the Xpert Carba-R. The sample preparation needs 1 minute and less than an hour for the run. The sensitivity and specificity of the Xpert Carba-R assay were higher compared with those of the reference culture and sequencing results (45).

\section{DNA Sequencing}

This method is used for the detection of carbapenemase genes and it can detect known and unknown genes that encode carbapenemases. In addition to that it can provide information about species and any other resistance genes. Because of its decreased cost, genome sequencing can now be applied in clinical microbiology laboratories. Metagenome sequencing uses DNA extracted directly from biological specimens instead of pure culture DNA which was used in whole-genome sequencing, (46).

\section{Current resistance status}

Although carbapenem resistance genes were documented among several countries in Asia, South America, and Europe, the situation in sub-Saharan Africa is not well documented (47).

The first strain of CRE was discovered in the 1980s after that it had been rapidly spread worldwide (48). Epidemiology studies suggest that there is a different geographic distribution for carbapenemases and specific carbapenemases prevalent in different parts of the world. For example, NDM-1 is prevalent in Pakistan and India, KPC is widespread in Italy, Greece, Colombia, Argentina, and United States while OXA-48 is endemic in North Africa, Turkey, the Middle-East and Malta (49).

Egypt is one of the first countries in the Eastern Mediterranean Region to establish a national surveillance system for health-care-associated infection (HAI). Egypt's HAI surveillance system that was established in May 2011, aimed to determine the prevalence rate and the incidence rate for HAI and to describe pathogens responsible for HAI to tell prevention and infection control activities (50, 51). There was an increase in the percentage of HAI cases due to CRE in Egypt between 2011 and 2017 (52) as from 3109 patients, 3836 Enterobacteriaceae isolates were reported to the surveillance system for HAI in Egypt and according to isolatelevel analysis, from 2306 isolates that were delivered to the reference laboratory, 1105 with a percentage of $47.9 \%$ were CRE which mean that about half $(47.9 \%)$ of the isolates were CRE (52). This is higher than measures stated in other Arab, Asian or African countries $(\mathbf{5 3}, \mathbf{5 4}, \mathbf{5 5}, \mathbf{5 6})$. The incidence of all CRE (HAI and non-HAI) in United States, Canada and China was 0.1-0.4/10,000 patient-days, 0.2 per 10,000 patient-days and 0.4 per 10,000 patient-days respectively which is much 
lower than the incidence of CRE HAI reported from Egypt(3.7/10,000 patientdays) $(\mathbf{5 7}, \mathbf{5 8}, \mathbf{5 9})$.

Therefore, it is important to implement strict infection control measures to prevent further dissemination of CRE in Egypt (52).

\section{References}

1. Suay-García B, Pérez-Gracia MT (2019). Present and Future of Carbapenemresistant Enterobacteriaceae (CRE) Infections Antibiotics (Basel).;19;8(3).

2. Lutgring D (2019). Carbapenemresistant Enterobacteriaceae: An emerging bacterial threat. Seminars in Diagnostic Pathology Volume 36, Issue 3, Pages 182-186.

3. Hawkey P, Livermore D (2012). Carbapenem antibiotics for serious infections. Br. Med. J.;344:e3236.

4. Patel G, Bonomo R (2013). 'Stormy waters ahead': Global emergence of carbapenemases. Front. Microbiol. ;4:48

5. Nordmann $\mathbf{P}$, Dortet $\mathbf{L}$, Poirel $\mathbf{L}$, (2012). Rapid detection of carbapenemase-producing

Enterobacteriaceae. Emerg Infect Dis; 18: 1503-7.

6. Breslow M (2014): "Illinois "Nightmare Bacteria" Outbreak Raises Alarms". PBS.org.

7. Centers for Disease Control and Prevention (2013). Action needed now to halt spread of deadly bacteria: Data show more inpatients suffering infections from bacteria resistant to all or nearly all antibiotics" (Press release). The Centers for Disease Control. March 5, 2013. Retrieved March 5, 2013. "During just the first half of 2012, almost 200 hospitals and long-term acute-care facilities treated at least one patient infected with these bacteria."

8.Dai W, Sun S, Yang P, et al. (2013). Characterization of carbapenemases extended-spectrum $\beta$-lactamases and molecular epidemiology of carbapenemnon-susceptible Enterobacter Cloacae in a Chinese hospital in Chongqing. Infect. Genet. Evol. 14:1-7.
9. Fernández J, Montero I, Martínez Ó, et al. (2015). Dissemination of multiresistantEnterobacter Cloacae isolates producing OXA-48 and CTXM-15 in a Spanish hospital. Int. J. Antimicrob. Agents 46, 469-74.

10.Liu C, Qin S, Xu H, et al. (2015). New Delhi metallo- $\beta$-lactamase 1 (NDM-1), the dominant carbapenemase detected in carbapenem-resistant Enterobacter Cloacae from Henan province, China. PLoS ONE 10:1371.

11.Sidjabat HE, Townell N, Nimmo GR, et al. (2015). The dominance of IMP-4producing Enterobacter Cloacae among carbapenemase-producing

Enterobacteriaceae in Australia. Antimicrob. Agents Chemother. 59, 4059-66.

12. Yang Q, Wang H, Sun H, et al. (2010). Phenotypic and genotypic characterization of Enterobacteriaceae with decreased susceptibility to carbapenems: results from large hospital-based surveillance studies in China. Antimicrob. Agents Chemother. 54:573-7.

13.Haidar G, Clancy CJ, Chen L, et al. (2017). Identifying Spectra of Activity and Therapeutic Niches for CeftazidimeAvibactam and Imipenem-Relebactam against Carbapenem-Resistant Enterobacteriaceae. Antimicrob Agents Chemother.; 61(9).

14.Little ML, Qin X, Zerr DM, et al. (2012).Molecular diversity in mechanisms of carbapenem resistance in paediatricEnterobacteriaceae.

International Journal of Antimicrobial Agents. 39 (1): 52-7.

15.Sho T, Muratani T, Hamasuna R, et al. (2013). The Mechanism of HighLevel Carbapenem Resistance in Klebsiellapneumoniae: Underlying Ompk36-Deficient Strains Represent a Threat of Emerging High-Level Carbapenem-Resistant K. pneumoniae with IMP-1 $\beta$-Lactamase Production in Japan. Microbial Drug Resistance. 19 (4).

16.Shin SY, Bae IK, Kim J, et al. (2012). Resistance to carbapenems in sequence type 11 Klebsiella pneumoniae is related to DHA-1 and loss of OmpK35 and/or 
OmpK36. Journal of Medical Microbiology. 61 (Pt 2):239-45.

17.Lutgring JD, Limbago BM (2016).

The Problem of Carbapenemase-

Producing-Carbapenem-Resistant-

Enterobacteriaceae Detection. J Clin

Microbiol.; 54(3):529-34.

18.Ambler $\mathbf{R}$ (1980). The structure of beta-lactamases. Philos. Trans. R. Soc. Lond. B Biol. Sci. 289 321-331.

19.Nordmann P, Naas T, Poirel L (2011). Global spread of Carbapenemaseproducing Enterobacteriaceae. Emerg Infect Dis.; 17(10):1791-8.

20.Bedenić B, Plečko V, Sardelić S, et al. (2014). Carbapenemases in gramnegative bacteria: laboratory detection and clinical significance. Biomed Res Int.; 2014:841951.

21.PfeiferY, Angela C, Wolfgang W (2010). Resistance to cephalosporins and carbapenems in Gram-negative bacterial pathogens. Int J Med Microbiol. 300 (6): 371-9.

22.Abdallah M, Balshi A (2018). First literature review of carbapenem-resistant $<\mathrm{i}>$ Providencia $</ \mathrm{i}>$. New Microbes New Infect.; 25:16-23.

23.Fernández J, Guerra B, Rodicio MR (2018). Resistance to Carbapenems in Non-Typhoidal Salmonella enterica Serovars from Humans, Animals and Food.Vet Sci.; 5(2).

24. Queenan A, Bush K (2007). Carbapenemases: The versatile $\beta$ lactamases. Clin. Microbiol. Rev.;20:440-458.

25.Paton R, Miles RS, Hood J, et al. (1993). ARI 1: beta-lactamase-mediated imipenem resistance in Acinetobacter baumannii. Int J Antimicrob Agents.; 2(2):81-7.

26.Mathers AJ, Hazen KC, Carroll J, et al. (2013). First clinical cases of OXA48-producing carbapenem-resistant Klebsiella pneumoniae in the United States: the "menace" arrives in the new world. J Clin Microbiol.; 51(2):680-3.

27. Choi JP, Cho EH, Lee SJ, et al. (2012). Influx of multidrug-resistant, Gram-negative bacteria (MDRGNB) in a public hospital among elderly patients from long-term care facilities: a singlecenter pilot study. Archives of
Gerontology and Geriatrics. 54 (MarchApril): 19-22.

28.Savard P, Perl TM (2012). A call for action: managing the emergence of multidrug-resistant Enterobacteriaceae in acute care settings. Current Opinion in Infectious Diseases. 25(4): 371-7.

29. Chitnis AS, Caruthers PS, Rao AK, et al. (2012). Outbreak of carbapenemresistant Enterobacteriaceae at a longterm acute care hospital: Sustained reductions in transmission through active surveillance and targeted interventions. Infection Control and Hospital Epidemiology. 33 (10): 98492.

30.Li J, Li Y, Song N, et al. (2019). Risk factors for carbapenem-resistant Klebsiella pneumonia infection: a metaanalysis. J Glob Antimicrob Resist. (19):30239-5.

31.Gupta N, Limbago BM, Patel JB, et al. (2011). Carbapenem-Resistant Enterobacteriaceae: Epidemiology and Prevention. Clinical Infectious Diseases. 53 (1): 60-7.

32.Landman D, Babu E, Shah N, et al. (2012). Transmission of carbapenemresistant pathogens in New York City hospitals: progress and frustration". Journal of Antimicrobial Chemotherapy. 67 (6): 1427-31.

33.Anderson KF, Lonsway DR, Rasheed JK, et al. (2007): Evaluation of methods to identify the Klebsiella pneumoniae carbapenemase in Enterobacteriaceae. J ClinMicrobiol; 45:2723-5.

34.Doyle D, Peirano G, Lascols C, et al (2012). Laboratory detection of enterobacteriaceae that produce carbapenemase. J ClinMicrobiol; 50: 3877-80.

35.Van der Zwaluw K, deHaan A, Pluister GN, et al. (2015). The carbapenem inactivation method (CIM), a simple and low-cost alternative for the Carba NP test to assess phenotypic carbapenemase activity in gram-negative rods. PLoS One; 10:e0123690.

36. Tijet N, Patel SN, Melano RG (2016). Detection of carbapenemase activity in Enterobacteriaceae: comparison of the carbapenem inactivation method versus 
the Carba NP test. J Antimicrobhemother;71:274-6.

37.Meini MR, Llarrull LI, Vila AJ (2015). Overcoming differences: the catalytic mechanism of metallo- $\beta$ lactamases. FEBS Lett; 589: 3419-32.

38.Franklin C, Liolios L, Peleg A (2006). Phenotypic detection of carbapenemsusceptible metallo-beta-lactamaseproducing gram-negative bacilli in the clinical laboratory. J ClinMicrobiol. 44(9):3139-44.

39.Arakawa Y, Shibata N, Shibayama K, et al. (2000): Convenient test for screening metallo- $\beta$-lactamaseproducing gram-negative bacteria by using thiol compounds. J ClinMicrobiol; 38:40-43.

40.Doi Y, Potoski BA, Adams-Haduch JM, et al. (2008). Simple Disk-Based Method for Detection of Klebsiella pneumonia Carbapenemase-Type $\beta$ Lactamase by Use of a Boronic Acid Compound. J ClinMicrobiol; 46:4083-6.

41.Tijet N, Boyd D, Patel SN, et al. (2013). Evaluation of the Carba NP test for rapid detection of carbapenemaseproducing Enterobacteriaceae and Pseudomonas aeruginosa. Antimicrob Agents Chemother; 57:4578-80.

42.Ledeboer NA, Lopansri BK, Dhiman $\mathbf{N}$, et al. (2015). Identification of gramnegative bacteria and genetic resistance determinants from positive blood culture broths by use of the Verigene Gramnegative blood culture multiplex microarray-based molecular assay. J ClinMicrobiol; 53: 2460-72. (MALDITOF/MS). Int $\mathrm{J}$ Antimicrob Agents; 48:655-60.

43.Hill JT, Tran KD, Barton KL, et al. (2014). Evaluation of the nanosphereVerigene $\mathrm{BC}-\mathrm{GN}$ assay for direct identification of gram-negative bacilli and antibiotic resistance markers from positive blood cultures and potential impact for more-rapid antibiotic interventions. J ClinMicrobiol; 52:3805-7.

44.Salimnia H, Fairfax MR, Lephart PR, et al. (2016). Evaluation of the FilmArray blood culture identification panel: results of a multicenter controlled trial. J ClinMicrobiol; 54:687-98.
45.Tato M, Ruiz-Garbajosa P, Traczewski M, et al. (2016). Multisite evaluation of Cepheid XpertCarba-R assay for detection of carbapenemaseproducing organisms in rectal swabs. J ClinMicrobiol; 54:1814-9.

46.Patel $\mathbf{R}$ (2016). New developments in clinical bacteriology laboratories. Mayo ClinProc ;91:1448-59.

47.Codjoe F, Donkor E (2018). Carbapenem Resistance: A review. Med. Sci. ;6:1.

48.Ito H, Arakawa Y, Oshuka S, et al. (1995). Plasmid-mediated dissemination of the metallo-beta-lactamase gene blaIMP among clinically isolated strains of Serratia marcescens. J. Antimicrob. Chemother. 50:503-511.

49.Nordmann P, Poirel L (2014). The difficult-to-control spread of carbapenemaseproducers

among Enterobacteriaceae worldwide. Clin. Microbiol. Infect.20:821-830.

50.See I, Lessa F (2013). Incidence and pathogen distribution of healthcareassociated infections in pilot hospitals in Egypt. Infect Control Hosp Epidemiol.;34:1281-8.

51.Talaat $M$, et al. (2016). National surveillance of health care-associated infections in Egypt: developing a sustainable program in a resourcelimited country. Am J Infect Control.;44:1296-301.

52.Kotb, S, Lyman M, Ismail G, et al. (2020). Epidemiology of Carbapenem-resistant

Enterobacteriaceae in Egyptian intensive care units using National Healthcareassociated Infections Surveillance Data,2011-2017. Antimicrob Resist Infect Control 9, 2.

53.Xu Y, Gu B, Hunag M, et al. (2015). Epidemiology of Carbapenem resistant Enterobacteriaceae (CRE). J Thorac Dis.; 7:376-85.

54.Mitgang EA, Hartley DM, Malchione MD, et al. (2018). Review and mapping of carbapenem-resistant Enterobacteriaceae in Africa: using diverse data to inform surveillance.

55.Moghnieh RA, Kanafani ZA, Tabaja HZ, et al. (2018). Epidemiology of common resistant bacterial pathogens in 
the countries of the Arab League. Lancet; Published online , https://doi.org/10.1016/S14733099(18)30414-6.

56.Ssekatawa K, Byarugaba DK, Wampande E, et al. (2018). A systematic review: the current status of carbapenem resistance in East Africa. BMC Res Notes.;11(1):629.

57.Brenner DJ, Krieg NR, Staley JT, et al. (2005). Bergey's Manual of Systematic Bacteriology ( $2^{\text {nd }}$ ed.). New York: Springer: 1108.

58. Thaden JT, Lewis SS, Hazen KC, et al. (2014). Rising rates of Carbapenem- resistant Enterobacteriaceae in community hospitals: A mixed-methods review of epidemiology and microbiology practices in a network of community hospitals in the southeastern United States. Infect Control Hosp Epidemiol. 35(8):978-83.

59.Mataseje LF, Abdesselam K, Vachon J, et al.( 2016). Results from the Canadian Nosocomial Infection Surveillance Program on Carbapenemase-Producing Enterobacteriaceae, 2010 to 2014. Antimicrob Agents Chemother.;60(11):6787-94. 